\title{
ANÁLISIS COMPARATIVO DE GUÍAS PARA EL DESARROLLO WEB ACCESIBLE
}

\section{COMPARATIVE ANALYSIS OF GUIDES FOR ACCESSIBLE WEB DEVELOPMENT}

\author{
Luis Felipe Londoño Rojas* \\ Valentina Tabares Morales* \\ Marta Rosecler Bez ${ }^{* *}$ \\ Néstor Darío Duque Mendez ${ }^{* * *}$ \\ Fecha de recepción: 22 de diciembre de 2016 \\ Fecha de revisión: 23 de julio de 2017 \\ Fecha de aprobación: 22 de septiembre de 2017
}

Cómo citar: L. F. Londoño Rojas, V. Tabares Morales, M. Rosecler Bez y N. D. Duque Mendez, "Análisis comparativo de guías para el desarrollo web accesible," Ciencia e Ingeniería Neogranadina, vol. 28, no. 1, pp. 101-115. DOI: http://dx.doi.org/10.18359/rcin.2683

\section{RESUMEN}

En la actualidad los sitios web no cumplen con requerimientos mínimos de accesibilidad, lo que impide que cualquier persona sin importar sus condiciones pueda acceder a los contenidos y servicios disponibles a través de estos sitios. Este problema se da en parte porque los desarrolladores no tienen conocimientos de los criterios y pautas de accesibilidad existentes o no saben cómo implementarlas. Como una alternativa, en este trabajo se presenta un

\footnotetext{
* Administrador de Sistemas Informáticos, estudiante del Departamento de Informática y Computación, Universidad Nacional de Colombia, Manizales, Colombia. Correo electrónico: Iflondonor@unal.edu.co. ORCID: https://orcid.org/0000-0002-9580-3898

** Administradora de Sistemas Informáticos y magíster en Ingeniería de Sistemas. Docente del Departamento de Informática y Computación, Universidad Nacional de Colombia, Manizales, Colombia. Correo electrónico: vtabaresm@unal.edu.co. ORCID: https://orcid.org/0000-0002-8078-8525

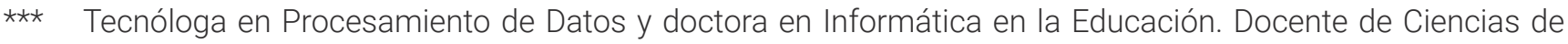
la Computación, Universidad Feevale, Novo Hamburgo, Brasil. Correo electrónico: martabez@feevale.br, martabez@gmail.com. ORCID: http://orcid.org/0000-0002-5542-8229

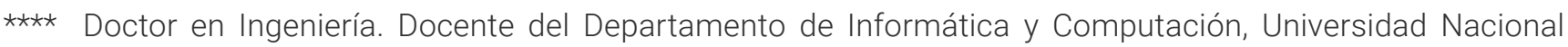
de Colombia, Manizales, Colombia. Correo electrónico: ndduqueme@unal.edu.co. ORCID: http://orcid. org/0000-0002-4608-281X
} 
análisis de guías para el desarrollo accesible, donde se evalúan diferentes criterios como las discapacidades que son atendidas, el nivel de conocimientos técnico que se requiere para su comprensión y la forma como se presentan los contenidos. A partir de esta evaluación se genera un cuadro comparativo y se hace la selección de las guías que pueden ser de mayor utilidad en la creación de sitios web accesibles, lo que puede servir como un apoyo a los desarrolladores al decidir cuál guía utilizar.

Palabras clave: accesibilidad, accesibilidad web, desarrollo accesible, guías de accesibilidad.

\section{ABSTRACT}

Currently, the websites do not meet minimum accessibility requirements, which prevents anyone, regardless of their conditions, from accessing the contents and services available through these sites. This problem is, partly because developers are not aware of existing accessibility criteria and guidelines or do not know how to implement them. As an alternative, this article presents an analysis of guideslines for approachable development, which assesses different criteria like the disabilities that are addressed, the level of technical knowledge required to understand the guides and the way the contents are presented. From this evaluation, a comparative table is generated, and the selection of the guides that can be most useful in the creation of accessible websites is made, which can serve as a support to the developers when deciding which guide to use.

Keywords: Accessibility, web accessibility, approachable development, accessibility guidelines.

\section{INTRODUCCIÓN}

La accesibilidad es una característica que representa la facilidad que se ofrece para que una persona pueda acceder a cualquier contenido u objeto, sin importar sus condiciones físicas, sociales, psicológicas, educativas o culturales [1]. Particularmente, en los sitios web, la accesibilidad es un factor importante para la inclusión de personas con discapacidades frente al mundo de la información y de las tecnologías. En este caso, la accesibilidad es entendida como el encuentro entre la capacidad funcional de una persona y las demandas de diseño del entorno digital que desea utilizar [2], [3].
Existen diferentes estándares para el desarrollo de sitios web accesibles, como las Web Content Accessibility Guidelines (WCAG 2.0), la Section 508 de Estados Unidos y otras normas nacionales. Sin embargo, en trabajos como los de [4]-[6] se ha identificado que la accesibilidad de los sitios web no llega a un nivel de cumplimiento aceptable de los criterios definidos en estas pautas y estándares internacionales. Eso impide que una persona en situación de discapacidad pueda navegar por los sitios web de manera fluida y natural.

Esta problemática se debe, en parte, a que los diseñadores de sitios web no tienen en 
consideración grupos especiales de personas con discapacidad como usuarios objetivo y han hecho poco esfuerzo para mejorar la accesibilidad, y también a que los desarrolladores no reconocen los requerimientos mínimos de accesibilidad o la forma como son presentados y estructurados no facilitan su entendimiento e incorporación [7]. Ante esto surgen como alternativa algunas guías prácticas que intentan facilitar la comprensión de los contenidos de los estándares de accesibilidad para que los desarrolladores los puedan comprender y aplicar en la creación de sus sitios web [8], [9].

No obstante, al existir más de una guía práctica para facilitar la comprensión del contenido de los estándares de accesibilidad, los desarrolladores encuentran dificultad a la hora de decidir en cuál de ellas basarse. Por esta razón, se presenta un análisis comparativo de diferentes guías prácticas disponibles en la web de acuerdo con tres criterios definidos, y se genera un cuadro con los resultados del análisis de las guías de accesibilidad que servirá a los desarrolladores para determinar cuál de ellas utilizar.

Este artículo presenta en la sección 1 el marco teórico que soporta el análisis realizado; en la sección 2 se listan las guías prácticas de desarrollo accesible que se van a analizar y evaluar; en la sección 3 se muestra la propuesta planteada en el artículo para llevar acabo el análisis de las guías para el desarrollo accesible; en la 4 se presentan los resultados obtenidos de la evaluación de las guías de acuerdo con la propuesta planteada en la sección anterior y también se muestra el análisis de los resultados obtenidos de la evaluación de las guías; la 5 se expone las conclusiones y trabajos futuros que se obtuvieron de este trabajo y la sección 6 , las referencias usadas en el trabajo.

\section{MARCO TEÓRICO Y TRABAJOS RELACIONADOS}

A continuación se explican algunos conceptos afines con la temática del artículo y la revisión de algunos trabajos relacionados.

La accesibilidad web es un concepto que hace referencia al conjunto de elemento que permite que una persona, sin importar sus condiciones, pueda acceder a los contenidos ofrecidos en la web [10]. Un sitio web es accesible cuando desde su diseño se han tenido en cuenta las diferencias existentes a nivel de habilidades y tecnologías de los usuarios que deseen acceder, si se han considerado las características físicas y cognitivas de los usuarios para evitar que se presenten barreras que puedan convertirse en un factor de exclusión [11].

Los estándares de la Section 508 son parte de la Federal Acquisition Regulation (FAR) y están orientadas al acceso electrónico para las personas con discapacidades físicas, sensoriales o cognitivas. Esta norma de accesibilidad es creada y utilizada en Estados Unidos de América; contiene criterios técnicos específicos para los distintos tipos de tecnologías basados en el rendimiento de los productos y se centra en las capacidades funcionales. Por esto, el software y aplicaciones deben cumplir con requerimientos funcionales que permitan el uso de los productos por parte de cualquier usuario, sin importar si este presenta algún tipo de limitación [12]-[14]. 
Las WCAG son los documentos de recomendaciones y pautas de accesibilidad para promover el desarrollo de sitios web accesibles creados por el World Wide Web Consortium (W3C). Las WCAG están en la versión 2.0, la cual se publicó en 2008 , y contiene 4 principios (perceptible, operable, comprensible y robusto), 12 pautas y 61 criterios, los cuales están orientados a lograr que el contenido web sea comprensible para el mayor número de personas con discapacidades posible [15], [16].

EI W3C ha creado otras guías para mejorar la accesibilidad de los sitios web, como son la Authoring Tool Accessibility Guidelines (ATAG) y la User Agent Accessibility Guidelines (UAAG); ambas actualmente están en su versión 2.0. Sin embargo, las WCAG son la guías más comunes para el desarrollo accesible y establecen las bases de normas de accesibilidad a nivel mundial [17].

Respecto a trabajos relacionados identificados en la literatura, algunos están enfocados a solucionar problemas específicos de accesibilidad web y otros realizan un análisis de guías de accesibilidad web existentes.

En el trabajo presentado en [18] se muestra una plataforma llamada Social4all, la cual realiza adaptaciones de un sitio web de acuerdo con el perfil del usuario con el objetivo de mejorar su accesibilidad. Igualmente, en [19] se plantea la adaptación como una alternativa para mejorar la accesibilidad de los sitios web, profundizando en la accesibilidad para personas con discapacidad auditiva, proponiendo como solución la adaptación sign-scripting, que es un mecanismo que representa gráficamente el lenguaje de señas. En [20] se presentan diferentes mecanismos para realizar adaptaciones de los sitios web para facilitar el acceso de personas con discapacidad auditiva, y de esta manera se muestra la importancia de las adaptaciones de lenguaje escrito a lenguaje de señas y su complejidad.

Por otro lado, en el trabajo presentado en [21] se realiza un estudio comparativo entre las WCAG 1.0 y las WCAG 2.0. Esta investigación se realizó con el objetivo de observar qué características de los sitios web de las empresas de Taiwán construidos siguiendo las WCAG 1.0 debían ser modificados para cumplir con las WCAG 2.0 y brindar un conjunto de recomendaciones para llevar a cabo este proceso de migración de la versión 1.0 a la 2.0. En [22] se lleva a cabo un análisis comparativo de las diferentes normas de accesibilidad de Estados Unidos con las de la OCDE y las de la Unión Europea con el fin de observar qué pautas de accesibilidad se aplican para personas mayores. Mientras que en el artículo [23] se presenta un estudio en el que se busca identificar los efectos en usuarios sin discapacidades y con discapacidades al implementar las directrices de accesibilidad dentro de un sitio web.

En la literatura se encuentran diferentes trabajos que realizan comparativas de normas y guías de accesibilidad; sin embargo, no se identificaron propuestas en las que se realizara un análisis de las guías prácticas de accesibilidad, teniendo en cuenta criterios para una mejor selección de estas.

\section{GUÍAS PARA EL DESARROLLO WEB ACCESIBLE}

Una guía para el desarrollo accesible es un conjunto de lineamientos de accesibilidad 
basados en normas y pautas reconocidas, que son presentados de diferentes formas para facilitar su comprensión y aplicación.

En la actualidad se encuentran disponibles diferentes herramientas con guías para el desarrollo de sitios web accesibles. A continuación, se listan algunas de estas identificadas al realizar revisión de literatura y en la web en general:

1. Developing Accessible Web Applications: esta herramienta fue desarrollada por Microsoft y está disponible en la dirección https://msdn.microsoft.com/en-us/ library/gg701991(v=vs.85).aspx. Los elementos HTML que contempla dentro de sus guías son enlaces, formularios, listas, tablas, encabezados e imágenes [24].

2. Accesibilidad Web: esta herramienta fue desarrollada por la Universidad de Alicante y cuenta con contenidos para comprender qué es la accesibilidad web. Está disponible en la dirección http:// accesibilidadweb.dlsi.ua.es/ [25].

3. IMS Guidelines for Developing Accessible Learning Applications: esta herramienta fue desarrollada por la IMS Global Learning Consortium. La herramienta es un repo-sitorio de guías de buenas prácticas de desarrollo y se encuentra disponible en la dirección https://www.imsglobal.org/accessibility/accessiblevers/index.html [26].

4. Developing Accessible Software Tutorial: esta herramienta fue desarrollada por la United States Access Board. Cuenta con tutoriales para la creación de software accesible según la Section 508 de Estados Unidos y se encuentra disponible en la dirección https://www. access-board.gov/guidelines-andstandards/communications-and-it/ about-the-section-508-standards/guideto-the-section-508-standards/developingaccessible-software-tutorial [27].

5. Web Accessibility Tutorials: esta herramienta fue desarrollada por el W3C y está disponible en la dirección https://www. w3.org/WAl/tutorials/. Cuenta con guías para el desarrollo de menús, imágenes, tablas y formularios accesibles [28].

6. Developing Accessible Websites: esta herramienta fue desarrollada por la Universidad de Washington y se encuentra disponible en la dirección http://www. washington.edu/accessibility/web/. Cuenta con guías accesibles para el desarrollo de formulario, imágenes, menús, tablas y encabezados [29].

7. Accesibilidad Web: esta herramienta fue desarrollada por la Sociedad Española de Documentación e Información Científica (SEDIC) como un curso para aprender conceptualmente qué es la accesibilidad web y se encuentra disponible en la dirección http://www.sedic.es/autoformacion/ accesibilidad/presentacion.html [30].

8. Prácticas de Web Accesibles: esta herramienta fue desarrollada por el Modelo de Acessibilidade de Governo Eletrônico (eMAG) de Brasil y está disponible en la dirección http://emag.governoeletronico.gov.br/cursodesenvolvedor/desenvolvimento-web/praticas-web-acessivel. html. Cuenta con guías para la creación de enlaces, imágenes, tablas, formularios, encabezados y contenidos multimedia accesibles [31]. 


\section{METODOLOGÍA PROPUESTA}

Se definió la realización de un análisis comparativo de las ocho guías para el desarrollo accesible que se presentaron en la sección anterior, ya que están disponibles en la web y son soportadas bajo los estándares de accesibilidad de la W3C, las WAI Guidelines (WCAG, ATAG, UAAG) y la Section 508.

El análisis se realiza con el fin de identificar las guías que mejor pueden ayudar a un desarrollador a aplicar las pautas de accesibilidad en los sitios web que construyen. Se definió una serie de criterios en los que se tiene en cuenta para cada guía la población beneficiaria, qué conocimientos previos se deben tener para comprenderlas y la forma como se muestran los contenidos.

A continuación, se presentan de forma detallada las características por evaluar de cada una de las guías para el desarrollo accesible identificadas:

1. Nombre de la guía: este campo servirá para identificar la guía y la organización que la desarrolló.

2. Tipo de producto: este campo es para identificar la forma como el sitio está proporcionando las guías, es decir, en forma de tutorial, cursos, talleres, página web con contenidos o alguna otra estrategia que aplique el sitio para mostrar las guías de accesibilidad.

3. Discapacidad: este criterio se subdivide en cuatro aspectos, que son equivalentes a los cuatro tipos de discapacidad reconocidas por las normas y estándares de accesibilidad, los cuales son discapacidad visual, auditiva, motriz y cogniti- vas. El criterio se utiliza para identificar si en la guía se presentan pautas para desarrollo accesible enfocadas a mejorar el acceso a personas que tengan alguna de las cuatro discapacidades. Por ejemplo, si se incluyen características como descripción de imágenes, textos alternativos y uso de comandos por teclado para la navegación, se indica que la guía incluye pautas para personas con discapacidad visual.

4. Nivel de tecnicidad requerido: este criterio tiene como objetivo identificar el nivel de detalle que tiene la guía analizada, con el fin de evaluar si requiere o no conocimientos avanzados, tanto en el área de desarrollo web, como en la de accesibilidad web. Para esto el criterio se divide en los siguientes subcriterios:

a. Alto: indica que la guía está orientada a personas con conocimientos superiores en el área de desarrollo web y accesibilidad web; es decir, una persona que se desenvuelve o trabaja directamente en el área de ciencias de la computación y por lo tanto posee conocimientos avanzados en lenguajes de programación web, análisis y desarrollo de software y otras cualidades que le facilitan la comprensión de una guía para desarrollo.

b. Medio: indica que la guía está encaminada para personas con conocimientos básicos en el área de desarrollo web; es decir, puede abarcar desde personas que están estudiando una carrera afín al área de ciencias de la computación, hasta 
personas que desarrollan por gusto propio. Estas últimas deben tener conocimientos básicos en lenguajes de programación web y una buena lógica computacional para así entender los contenidos de las guías de desarrollo.

c. Bajo: indica que la guía está destinada para cualquier persona, sin importar si tiene o no conocimientos sobre desarrollo web o accesibilidad web. Es decir, el nivel del contenido ofrecido por la guía no profundiza en temas de desarrollo más técnicos, como los lenguajes de programación web, el uso de etiquetas y las características del lenguaje, lo cual permite que una persona pueda entender más sobre qué es el desarrollo accesible, mas no cómo hacer desarrollo accesible.

5. Nivel del contenido: este criterio tiene como objetivo identificar qué tan amplio es el contenido proporcionado en la guía. Para esto el criterio se divide en los siguientes subcriterios:

a. Alto: indica que los contenidos de la guía son amplios y presentados de manera clara y concisa, lo cual hace que el lector los pueda entender fácilmente. Además, indica que los contenidos son presentados gráfica y textualmente, y ofrecen así ejemplos prácticos y actividades que faciliten el proceso de aprendizaje de la guía.

b. Medio: indica que los contenidos expuestos en la guía no son extensos; sin embargo, presentan claridad en su forma de ser presentados y ofrecen algunos mecanismos facilitadores para el entendimiento de la guía.

c. Bajo: indica que el contenido de la guía solo se presenta de manera textual y sus contenidos son de carácter conceptual descriptivo, lo cual no facilita el entendimiento de la guía y podría no atraer al usuario a volver a ver estos contenidos.

Una vez definidos los criterios para realizar el análisis comparativo de las guías para desarrollo web accesible se procede a su evaluación por etapas de la siguiente forma:

1. Etapa inicial: se ingresa a cada una de las herramientas y se realiza una revisión detallada de los diferentes campos y criterios definidos anteriormente.

2. Etapa intermedia: se construye y completa el cuadro comparativo de las herramientas con la información que se obtuvo en la anterior etapa.

3. Etapa final: se realiza un análisis de los resultados obtenidos de las herramientas para determinar cuáles de las guías pueden ser de mayor utilidad para un desarrollador. Para esta selección se tienen en cuenta los siguientes criterios:

a. Guías que atienden tres o más tipos de discapacidad.

b. Nivel de tecnicidad requerido medio o bajo.

c. Nivel del contenido alto. 


\section{RESULTADOS}

Como producto del proceso de comparación de acuerdo con los criterios presentados en la sección 3, se generó un cuadro comparativo que se presenta en la Tabla 1, ubicando en cada columna una $X$ para el caso en que sí se cumplía.

Tabla 1. Tabla comparativa de guías para el desarrollo de sitios web accesibles

\begin{tabular}{|c|c|c|c|c|c|c|c|c|c|c|c|}
\hline \multirow{2}{*}{ Nombre } & \multirow{2}{*}{$\begin{array}{l}\text { Tipo de } \\
\text { producto }\end{array}$} & \multicolumn{4}{|c|}{ Discapacidad } & \multicolumn{3}{|c|}{ Nivel de Tecnicidad } & \multicolumn{3}{|c|}{ Nivel del Contenido } \\
\hline & & Visual & Auditivo & Motrices & Cognitivas & Alto & Medio & Bajo & Alto & Medio & Bajo \\
\hline $\begin{array}{l}\text { Accesibilidad Web- } \\
\text { Universidad de } \\
\text { Alicante }\end{array}$ & $\begin{array}{l}\text { Pagina } \\
\text { Web }\end{array}$ & $x$ & & $x$ & $x$ & & $x$ & & & $x$ & \\
\hline $\begin{array}{c}\text { Developing accesible } \\
\text { Web Applications - } \\
\text { Miscrosoft }\end{array}$ & $\begin{array}{l}\text { Pagina } \\
\text { Web }\end{array}$ & $x$ & $x$ & $x$ & $x$ & & $x$ & & $x$ & & \\
\hline $\begin{array}{l}\text { IMS Guidelines for } \\
\text { Developing Accesible } \\
\text { Learning Applications } \\
\text { - IMS Global Learning } \\
\text { Consortium }\end{array}$ & $\begin{array}{l}\text { Pagina } \\
\text { Web }\end{array}$ & $x$ & $x$ & $x$ & $x$ & & $x$ & & & $x$ & \\
\hline $\begin{array}{c}\text { Developing } \\
\text { Accesible Websites } \\
\text { - Universidad de } \\
\text { Washington }\end{array}$ & $\begin{array}{l}\text { Pagina } \\
\text { Web }\end{array}$ & $x$ & $x$ & $\mathrm{x}$ & & & $x$ & & $\mathrm{x}$ & & \\
\hline $\begin{array}{c}\text { Developing Accesible } \\
\text { Software Tutorial - } \\
\text { United States Access } \\
\text { Board }\end{array}$ & $\begin{array}{l}\text { Pagina } \\
\text { Web }\end{array}$ & $x$ & $x$ & $x$ & $x$ & $x$ & & & & $x$ & \\
\hline $\begin{array}{l}\text { Web Accessibility } \\
\text { Tutorials - W3C }\end{array}$ & $\begin{array}{l}\text { Tutorial } \\
\text { Web }\end{array}$ & $x$ & $x$ & $x$ & $x$ & & X & & $x$ & & \\
\hline $\begin{array}{c}\text { Pràcticas de } \\
\text { accesibilidad web - } \\
\text { eMAG gobierno de } \\
\text { Brasil }\end{array}$ & $\begin{array}{l}\text { Tutorial } \\
\text { Web }\end{array}$ & $x$ & $x$ & $x$ & $x$ & & $x$ & & $x$ & & \\
\hline $\begin{array}{l}\text { Accesibildad Web - } \\
\text { SEDIC }\end{array}$ & $\begin{array}{l}\text { Curso } \\
\text { Online }\end{array}$ & $x$ & & $x$ & & & & $x$ & & $x$ & \\
\hline
\end{tabular}

Fuente: elaboración propia.

En la Fig. 1 se muestra el resultado de la evaluación para todas las guías, bajo el criterio de discapacidad, donde se puede identificar que la mayoría de las pautas para el desarrollo accesible de sitios web está orientada a personas con discapacidad visual y motriz. Esto indica que todas las guías analizadas ofrecen contenidos para el desarrollador, con el fin de que sus sitios web tengan elementos como textos alternativos, descripciones de botones, tablas e imágenes y otros elementos de la estructura de la página. De la misma manera, comandos por teclado que faciliten tanto el uso de un lector de pantalla, como minimizar la interacción del usuario con el hardware del computador, lo que permite a una persona con alguna de estas discapacidades navegar fluidamente por el sitio web. 


\section{Ciencia e Ingeniería Neogranadina}

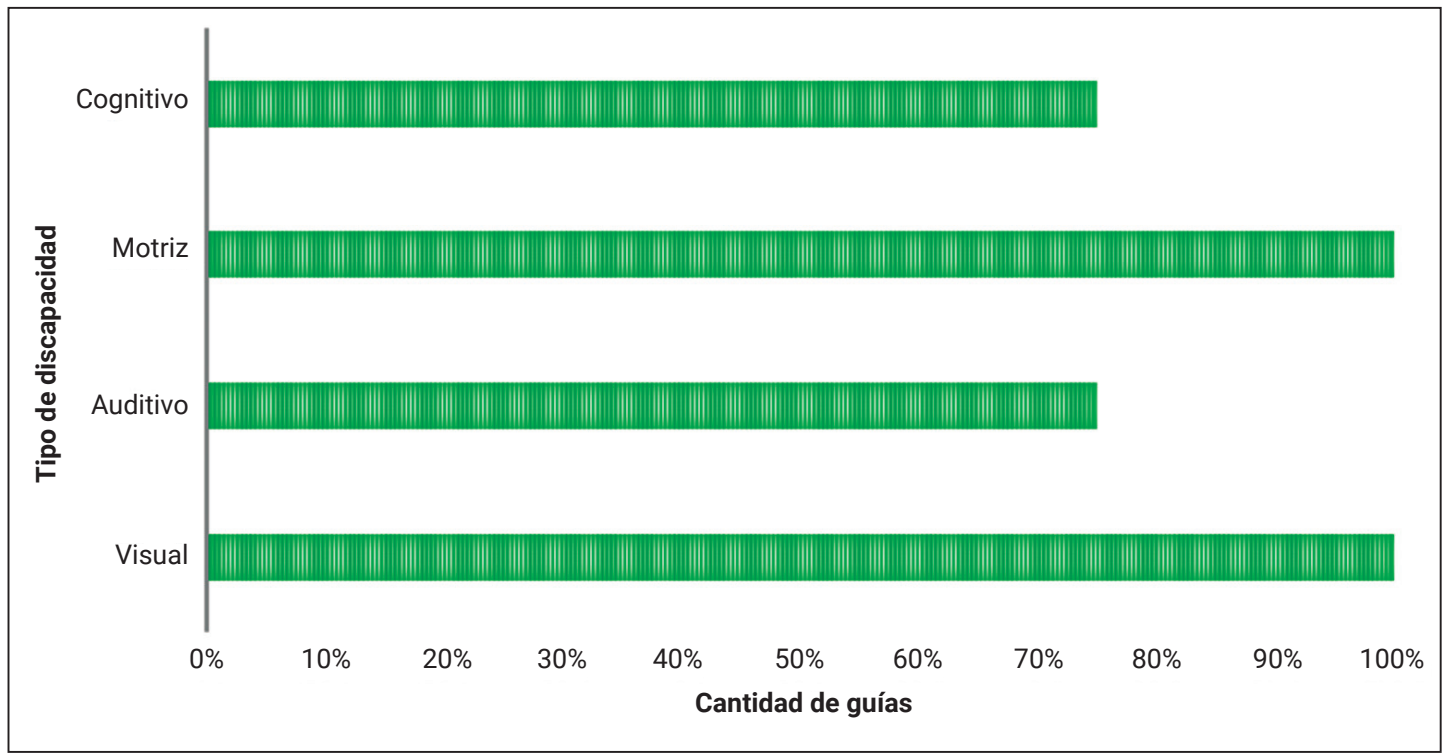

Fig. 1. Discapacidades analizadas

Fuente: elaboración propia.

En la Fig. 2 se observa que el contenido ofre- de accesibilidad web, ya que la mayor parte cido por las guías analizadas está orientado de las guías en el proceso de explicación de a personas con conocimientos básicos en las pautas definen también los conceptos de desarrollo. Esto indica que la persona no accesibilidad web e informan al usuario de necesariamente debe tener conocimientos estos y su importancia.

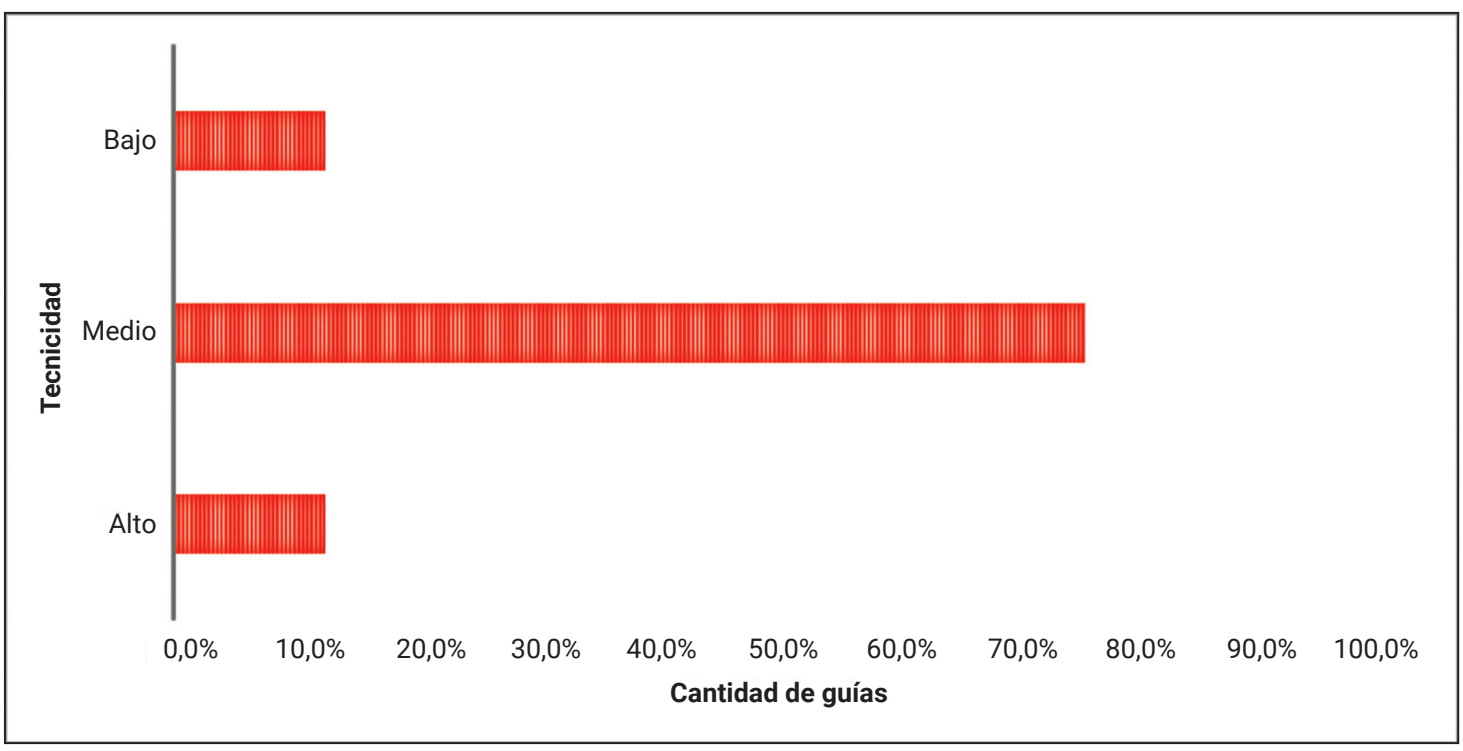

Fig. 2. Tecnicidad requerida de las guías

Fuente: elaboración propia. 
Como se observa en la Fig. 3, el nivel del contenido de los materiales ofrecidos por las guías es medio o alto, lo cual indica que la forma como las guías exponen sus contenidos es de fácil comprensión para los desarrolladores o personas que la vayan a utilizar.

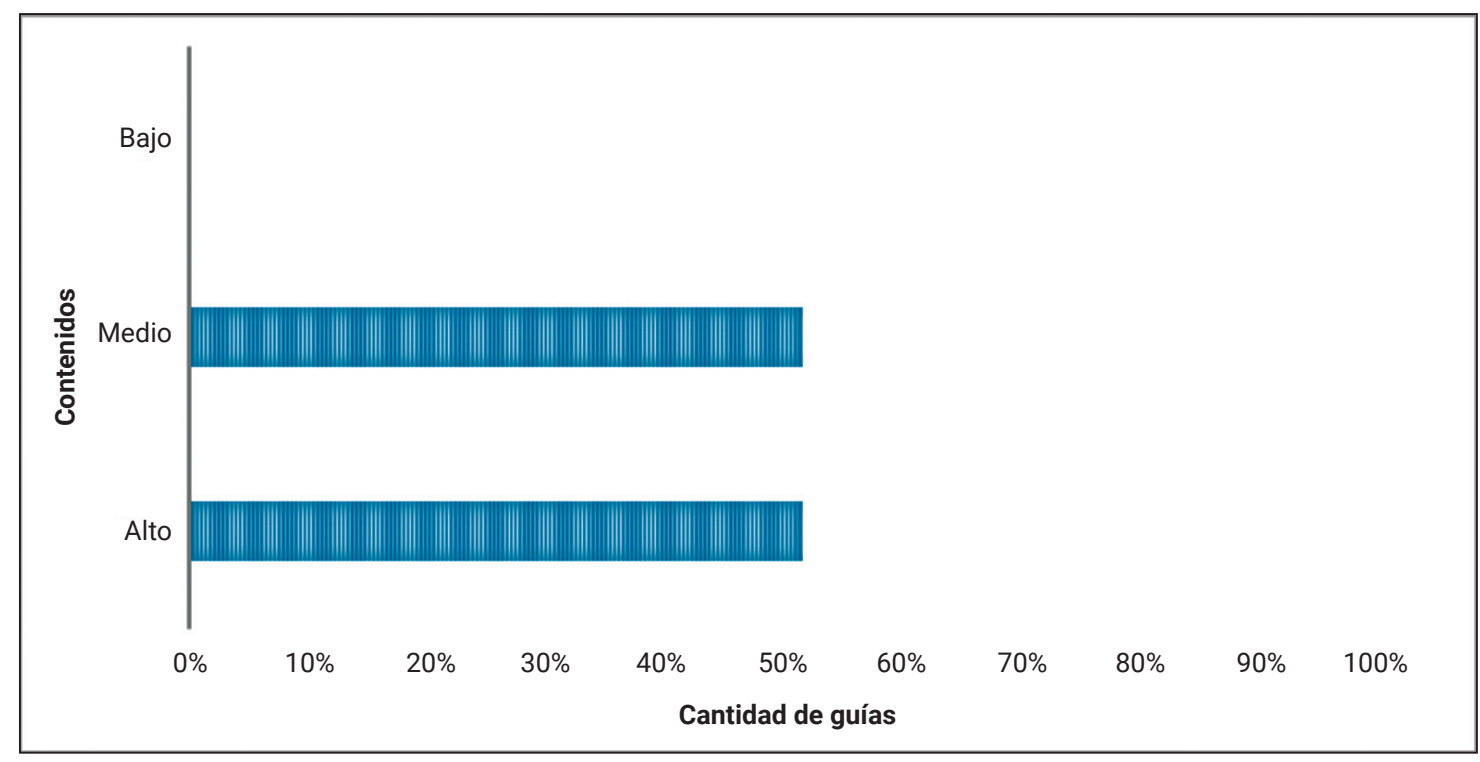

Fig. 3. Contenido de las guías

Fuente: elaboración propia.

Teniendo en cuenta los resultados presentados previamente, fueron seleccionadas las guías que cumplen con los siguientes criterios necesarios para proporcionar una mayor ayuda a los desarrolladores:

- Las guías presentadas deben atender tres o más tipos de discapacidad para así abarcar una población mayor y que se cubran más necesidades de acceso.

- El nivel de conocimientos técnicos requeridos por el usuario para comprender el material de las guías debe ser medio o bajo, para permitir facilidad en el entendimiento de las guías con el menor conocimiento posible sobre accesibilidad y desarrollo web.
- Finalmente, el nivel de los contenidos de las guías prácticas debe ser alto, lo cual permite más diversidad en la forma como se presenta el material en guías para que el usuario pueda adquirir el conocimiento y aplicarlo en sus desarrollos.

De acuerdo con lo anterior, al realizar la valoración del primer criterio, cumplieron 7 de las 8 guías. Finalizada la valoración del segundo criterio cumplieron 6 de las 7 guías restantes y al hacer la valoración con el tercer criterio, 4 de las 6 guías cumplían. Estas últimas fueron seleccionadas y se muestran a continuación:

- Web Accessibility Tutorials - W3C: esta guía es presentada en forma de tutorial y es un producto del W3C (World Wide Web 
Consortium). Está orientada para los tipos de discapacidad identificadas en los estándares de accesibilidad. El nivel de tecnicidad que requiere una persona para usarla es medio y el nivel de contenido, alto, por lo cual el desarrollador o persona interesada podrá tener material presentado de diferentes maneras y con información clara para facilitar su proceso de aprendizaje.

- Developing Accessible Web Applications - Miscrosoft: esta guía fue desarrollada por la compañía Microsoft y presenta características que pueden facilitar al desarrollador a entender y utilizar las pautas de desarrollo accesible. Sus contenidos son amplios, entendibles, se muestran de diferentes formas y el nivel de tecnicidad de las personas es medio. Además, las pautas que se suministran dentro de la guía están orientadas para mejorar la accesibilidad de personas que tengan cualquiera de los cuatro tipos de discapacidad definidos en los estándares de accesibilidad.

- Prácticas de accesibilidad web - eMAG Gobierno de Brasil: esta guía la desarrollo el Gobierno brasilero en el marco de la normativa eMAG para la creación de sitios web inclusivos. Las prácticas de accesibilidad que se ofrecen en esta guía son con base en las pautas de desarrollo accesible del W3C. Sus contenidos son claros y bastos, y las pautas ofrecidas son para mejorar la inclusión de personas con discapacidad visual, auditiva, motriz y cognitiva.

- Developing Accessible Websites - Universidad de Washington: esta guía fue crea- da por la Universidad de Washington y, al igual que la guía anterior, presenta varias características para facilitar su entendimiento y para que el desarrollador pueda hacer uso de las pautas. Sus contenidos son extensos, comprensibles, se presentan de diferentes formas y el nivel de tecnicidad de las personas es medio. Sin embargo, las pautas brindadas están enfocadas en facilitar la accesibilidad web de personas con discapacidad visual, auditiva y motriz, por lo cual los sitios que cree un desarrollador a partir de esta guía probablemente no serán accesible para una persona con algún tipo de discapacidad cognitiva.

\section{CONCLUSIONES Y TRABAJOS FUTUROS}

Las guías para el desarrollo accesible brindan pautas a los desarrolladores para que sus sitios sean navegables por personas que presentan discapacidad visual y motora. Un problema de estas guías es que a su vez no están brindando pautas para la discapacidad auditiva y cognitiva, aspecto que repercute en un problema tanto para el desarrollador, como para las personas con estas discapacidades.

Este trabajo puede servir como una guía para los desarrolladores al momento de decidir qué guía práctica de accesibilidad usar para crear sitios web. Se resalta que en los trabajos revisados en la literatura se realizan análisis comparativos de leyes de accesibilidad o de las versiones de la misma guía de accesibilidad, pero no comparación de guías de acuerdo con criterios como las 
comunidades que se tienen en cuenta, el nivel de conocimientos previos requeridos y la forma como se muestran los contenidos.

Las guías de desarrollo accesible evaluadas y seleccionadas presentan las pautas básicas para que los sitios web puedan ser accesibles. Se espera que aquellos sitios desarrollados, siguiendo estas guías, puedan alcanzar un mayor nivel de accesibilidad respecto a sitios que no contemplan estas características.

Al agrupar las guías prácticas por tipo de producto, podemos observar que cinco de estas que fueron analizadas son páginas web y que solo dos de ellas, según los criterios de evaluación, proporcionan una mayor ayuda a los desarrolladores. De las dos guías analizadas que son tutoriales web, ambas cumplieron con los criterios de evaluación. Por lo tanto, se puede decir que al exponer la información de los lineamientos de accesibilidad como tutorial (paso a paso) podrían llegar a ser más completas las explicaciones y facilitar así su comprensión.

Como trabajo futuro se plantea realizar el análisis de más guías de desarrollo accesible para ofrecer a los desarrolladores un mayor apoyo y que puedan incorporar mejores prácticas de accesibilidad en los sitios web construidos. Además, teniendo en cuenta que estas guías son para el desarrollo accesible, se espera realizar evaluación de accesibilidad web de los sitios donde se presentan, para validar que sí se estén aplicando las pautas que promueven. Finalmente, se está trabajando en la construcción de una herramienta que contenga un conjunto de guías prácticas para el desarrollo accesible basadas en las guías seleccionadas, donde además se haga validación de algunas secciones del código fuente y se ofrezca retroalimentación al desarrollador.

\section{AGRADECIMIENTOS}

El trabajo presentado en este artículo se enmarca en los proyectos Modelo orientado a la accesibilidad y adaptatividad en repositorios y federaciones de recursos educativos digitales, con código 35806 y Gestión de recursos educativos digitales accesibles, con código 28828, financiados por la Universidad Nacional de Colombia.

\section{REFERENCIAS}

[1] J. I. D. Tafur, C. M. V. White, C. R. Caicedo et al. Fundamentación conceptual para la atención en el servicio educativo a estudiantes con Necesidades Educativas Especiales (NEE). Bogota: Ministerio de Educación Nacional, 2006.

[2] L. F. Londoño, V. Tabares y N. Duque, "Evolución en la accesibilidad de la Federación de Repositorios de Objetos de Aprendizaje Colombia (FROAC)," en VII Congr. Int. Ambient Virtuales Aprendiz Adapt y Accesibles, 2015, pp. 1-11.

[3] R. Miñón, L. Moreno, P. Martínez y J. Abascal, "An approach to the integration of accessibility requirements into a user interface development method," Sci Comput Program, vol. 86, pp. 58-73, 2014. DOI: https://doi.org/10.1016/j. scico.2013.04.005 
[4] M. V. S. Figueroa, P. L. Alfonzo, S. I. Mariño y M. V. Godoy, "Evaluación de la Accesibilidad en Dos Sitios Bancarios Nacionales Dependientes de la Administración Pública," Rev. Latinoam Ing. Softw, vol. 2, no. 3, pp. 144-148, 2014. DOl: https://doi.org/10.18294/ relais.2014.144-148

[5] J. R. Hilera, L. Fernández, E. Suárez, E. T. Vilar, "Evaluación de la accesibilidad de páginas web de universidades españolas y extranjeras incluidas en rankings universitarios internacionales," Rev. Española Doc. Científica, vol. 36, no. 1, pp. 1-16.

[6] F. Iniesto y R. Covadonga, "Evaluación de la accesibilidad en las plataformas MOOC en español: UNED COMA, COLMENIA y Miriada X," en XVI Simp. Int. Informática Educ., 2014, pp. 233-327.

[7] P.-L. P. Rau, L. Zhou, N. Sun y R. Zhong, "Evaluation of web accessibility in China: changes from 2009 to 2013," Univers. Access. Inf Soc., vol. 15, pp. 297-303, 2016. DOI: https://doi. org/10.1007/s10209-014-0385-9

[8] H. L. Antonelli, R. P. Fortes, "A Support for Developers Implement the Accessibility Guidelines Regarding to Web Menus," en Proc 21st Brazilian Symp Multimed Web - WebMedia '15, 2015, pp. 33-40. DOI: https://doi. org/10.1145/2820426.2820445

[9] M. P. González, C. I. Chesñevar y R. Brena, "Human Interface and the Management of Information. Information and Knowledge Design," en HIMI: International Conference on Human Interface and the Management of Information, 2015, pp. 595-606. DOI: https://doi. org/10.1007/978-3-319-20612-7

[10] J. A. Martínez y N. P. Lara. La Accesibilidad de los Contenidos Web. BarceIona: UOC, 2007.

[11] C. H. G. Campo, "Herramientas Web 2.0 y accesibilidad a sitios Web para la apropiacion social de conocimiento en una ciudad educadora," Revista Universidad EAFIT, vol. 45, no. 153, pp. 26-37, 2009.

[12] P. J. Jaeger, "Beyond Section 508: The spectrum of legal requirements for accessible e-government Web sites in the United States," Jour. Gov. Inf., vol. 30, pp. 518-533, 2004. DOI: https://doi. org/10.1016/j.jgi.2004.09.010

[13] B. J. Thatcher, "Section 508 Web Standards and WCAG Priority 1 Checkpoints: A Side-by-Side Comparison," The Research Exchange, vol. 6, no. 3, pp. 1-12, 2001.

[14] B. Krepp, "Development of section 508-compliant document production protocols and websites," Transp. Res. Rec. vol. 2469, pp. 100-107, 2014. DOI: https://doi.org/10.3141/2469-11

[15] W3C, "Web Content Accessibility Guidelines (WCAG) 2.0," 2008. [En línea]. Dosponible en: https://www.w3.org/ TR/WCAG20/

[16] B. Kelly, D. Sloan, L. Phipps et al., "Forcing Standardization or Accommodating Diversity? A Framework for Applying the WCAG in the Real World," en Inter. Cross-Disciplinary 
Workshop on Web Accessibility, 2005, pp. 46-54. DOI: https://doi. org/10.1145/1061811.1061820

[17] D. R. Agüero y A. A. Perea, "Diseño de sitios web accesibles," 2009. [En línea]. Disponible en: http://www.virtualeduca. info/ponencias2010/8/AWUNLAR.pdf

[18] R. Gonzalez, J. Pascual, D. Burgos, "Social4all: Definition of specific adaptations in Web applications to improve accessibility," Computer Standards \& Interfaces, vol. 48, pp. 1-9, 2016. DOI: https://doi.org/10.1016/j. csi.2016.04.001

[19] R. González Crespo, L. Joyanes Aguilar y O. Sanjuán Martínez, "Improving access to IT services for people with disability through software aids," Journal Ambient Intell Smart Environ, vol. 4, no. 6, pp. 563-564, 2012. DOI: https:// doi.org/10.3233/AIS-2012-0181

[20] R. G. Crespo, O. S. Martínez, "La Web 3.0 al servicio de las personas discapacitadas auditivas mediante las pautas de accesibilidad 2.0," Sociedad y Utopía, no. 36, pp. 153-172, 2010.

[21] S. H. Li, D. C. Yen, W. H. Lu y T. L. Lin, "Migrating from WCAG 1.0 to WCAG 2.0 - A comparative study based on Web Content Accessibility Guidelines in Taiwan," Computers in Human Behavor, vol. 28, pp. 87-96, 2012. DOI: https:// doi.org/10.1016/j.chb.2011.08.014

[22] Y. T. Yang y B. Chen, "Web accessibility for older adults: A comparative analysis of disability laws," Gerontologist, vol. 55, pp. 854-864, 2015. DOI: https:// doi.org/10.1093/geront/gnv057
[23] S. Schmutz, A. Sonderegger y J. Sauer, "Implementing Recommendations From Web Accessibility Guidelines," Human Factors: The Journal of the Human Factors and Ergonomics Society, vol. 58, pp. 611-629, 2016. DOI: https://doi. org/10.1177/0018720816640962

[24] Microsoft Developing Accessible Web Applications, "Semantic HTML,". [En línea]. Disponible en: https:// msdn.microsoft.com/en-us/library/ gg701991(-v=vs.85).aspx\#name_ forms

[25] Universidad de Alicante, "Errores típicos," s. f. [En línea]. Disponible en: http:// accesibilidadweb.dlsi.ua.es/?-menu=ej-errorestipicos

[26] IMS Global Learning Consortium, "IMS Guidelines for Developing Accessible Learning Applications," [En línea]. Disponible en: https://www.imsglobal. org/accessibility/accessiblevers/index.html.

[27] Section 508, "Developing Accessible Software Tutorial," [En línea]. Disponible en: https://www.access-board. gov/guidelines-and-standards/ communications-and-it/about-thesection-508-standards/guide-tothe-section-508-standards/developing-accessible-software-tutorial.

[28] W3C Web Accessibility, "Tutorials," [En línea]. Disponible en: https://www. w3.org/WAl/tutorials/

[29] University of Washington Accessible Technology, s. f. [En línea]. Disponible en: http://www.washington.edu/accessibility/web/ 
[30] SEDIC, "Pautas de Accesibilidad al Contenido en la Web (WCAG)," [En línea]. Disponible en: https://www. sedic.es/autoformacion/accesibilidad/7-pautas-accesibilidad.html
[31] eMAG, "Práticas Web acessíveis," s. f. [Em línea]. http://emag.governoeletronico.gov.br/cursodesenvolvedor/desenvolvimento-web/praticas-web-acessivel.html 